\title{
Genomic Landscape and Targeted Treatment of Gallbladder Cancer: Results of a First Ongoing Prospective Study
}

\author{
Amol Patel ${ }^{1}$ Dharmesh Soneji ${ }^{1}$ Harinder Pal Singh ${ }^{1}$ Manish Kumar ${ }^{1}$ Arnab Bandyopadhyay ${ }^{2}$ \\ Ankit Mathur ${ }^{3}$ Anuj Sharma ${ }^{4}$ Gaurav Prakash Singh Gahlot ${ }^{5}$ Shivashankara MS ${ }^{1}$ \\ Bhupesh Guleria ${ }^{1}$ Rajesh Nair ${ }^{1}$ Dipen Bhuva' Suresh Pandalanghat ${ }^{1}$
}

\footnotetext{
${ }^{1}$ Department of Medical Oncology, Army Hospital Research and Referral, New Delhi, India

${ }^{2}$ Department of Surgical Oncology, Command Hospital (Eastern Command), Kolkata, West Bengal, India

${ }^{3}$ Department of Interventional Radiology, Army Hospital Research and Referral, New Delhi, India

${ }^{4}$ Department of Gastrointestinal Surgery, Army Hospital Research and Referral, New Delhi, India

${ }^{5}$ Department of Pathology, Army Hospital Research and Referral, New Delhi, India
}

South Asian J Cancer:2020;9:74-79

\begin{abstract}
Address for correspondence Suresh Pandalanghat, MD (Gen Med) DNB (Med Oncology) Department of Medical Oncology, Army Hospital Research and Referral, Dhaula Kuan, New Delhi 110010 , India (e-mail: psuresh_n@yahoo.com).
\end{abstract}

\begin{abstract}
Keywords

- comprehensive genomic profiling

$-E R B B 2 / 3$

- gallbladder cancer

- targeted therapy

Background Prognosis of gallbladder cancer $(G B C)$ has not changed in the past 20 years. Comprehensive genomic profiling (CGP) carries potential to determine the actionability for multiple targets, including ERBB2, ERBB3, MET, ROSI, FGFR, and PIK3. This study evaluates the role of CGP and targeted therapies.

Methods This is a multicenter, prospective, single-arm study. All consecutive patients of unresectable and/or metastatic GBC of age $\geq 18$ years were enrolled. Hybrid capture-based CGP was performed by Foundation Medicine CDx. All patients received first-line chemotherapy with gemcitabine-cisplatin regimen. Patients with ERBB2/3 amplification received trastuzumab with capecitabine or nab-paclitaxel, and patients with $M E T$ amplification were treated with crizotinib. For ERBB2/3 mutations, lapatinib plus capecitabine regimen was used.

Results Fifty patients were studied with a median age of 56 years (range 26-83) and a male-to-female ratio of 1:1.6. ERBB2 and ERBB3 amplification was seen in $9(18 \%)$ and 2 (4\%) patients, respectively. Four patients with ERBB2 amplification received trastuzumab and/or lapatinib, showed partial response, and maintained response beyond 12 weeks. One patient had mixed response, whereas two patients progressed on trastuzumab and lapatinib. Three patients with ERBB3 mutations showed response to lapatinib-capecitabine. One patient with MET amplification responded to crizotinib for 4 weeks. PIK3 mutations were present in $14 \%$ of cases and were independent of ERBB aberrations.

Conclusion GBC is enriched in $28 \%$ of patients with ERBB2 and ERBB3 amplifications and/or mutations. Responses are seen with lapatinib in concurrent ERBB2 mutation and amplification. ERBB3 mutation showed response to lapatinib. MET and PIK3 are new findings in $G B C$, which may be targeted.
\end{abstract}

DOI https://doi.org/ 10.1055/s-0040-1721180 ISSN 2278-330X. (c) 2020. Medlntel Services Pvt Ltd.

This is an open access article published by Thieme under the terms of the Creative Commons Attribution-NonDerivative-NonCommercial-License, permitting copying and reproduction so long as the original work is given appropriate credit. Contents may not be used for commercial purposes, or adapted, remixed, transformed or built upon. (https://creativecommons.org/licenses/by-nc-nd/4.0/)

Thieme Medical and Scientific Publishers Pvt. Ltd., A-12, 2nd Floor,

Sector 2, Noida-201301 UP, India 


\section{Introduction}

Gallbladder cancer (GBC) has peculiar geographic distribution; although it is rare in Northern America, it is one of the most common malignancies in North India, Pakistan, Bangladesh, Chile, Ecuador, Japan, and Korea. ${ }^{1-3}$ It is more common in females, and its anatomical location, presentation with obstructive jaundice, and chemotherapy refractoriness make it one of the aggressive malignancies, limiting median overall survival to 3 months in metastatic setting in untreated patients. Gemcitabine-cisplatin is the standard of care as per the meta-analysis of ABC02 and BT22 trials of advanced biliary cancers in the first-line setting, wherein majority of patients had cholangiocarcinoma. ${ }^{4}$ Survival in GBC has not improved over the past 20 years, ${ }^{5}$ highlighting the importance of newer therapies. In the era of personalized and precision medicine, we felt the need to conduct a prospective study of comprehensive genomic profiling (CGP) in advanced GBC to find tumor- and site-specific genomic alterations. The purpose was to find out driver mutation and amplification in GBC and treat these patients with available therapies in the absence of standard of care in the second-line setting.

\section{Methods}

\section{Patients}

The study was conducted at two tertiary care centers of Armed Forces. All consecutive patients of locally advanced unresectable and/or metastatic GBC were included. Fifty patients were enrolled from August 2018 after approval of the Institutional Ethical Committee. Diagnosis of GBC was made on the basis of imaging findings and was confirmed with biopsy and/or fine needle aspiration cytology. Patients aged $\geq 18$ years were eligible. For staging work-up, contrast-enhanced computed tomography (CT) of the chest, abdomen, and pelvis, or positron emission tomography CT of the whole body was performed. Patients were analyzed for baseline complete blood count, liver function tests, renal function tests, carbohydrate antigen (CA) 19-9 levels, and carcinoembryonic antigen (CEA) levels. Patients with serum bilirubin $\leq 3 \mathrm{mg} / \mathrm{dL}$ and aspartate transaminase/alanine aminotransferase up to three times of normal were permitted for enrollment. Patients of Eastern Cooperative Oncology Group (ECOG) Performance Status (PS) 3 and 4 were excluded. Demographic profile information was collected as per the prespecified protocol. Patients were excluded if they had intrahepatic or extrahepatic cholangiocarcinoma. ECOG method was used for assessing PS. ${ }^{6}$

\section{Biopsy}

Biopsies were performed under ultrasound guidance for all patients before start of chemotherapy. Laparoscopic biopsies were performed for patients who could not undergo ultrasound-guided biopsies. Laparoscopic biopsies were taken from local lesion and/or peritoneal deposits. Biopsy sample was preserved in $10 \%$ neutral-buffered formalin for 24 hours, and, subsequently, formalin-fixed paraffin-embedded blocks were made. These blocks were centrally collected and transported to a central facility in the United States. Biopsies were permitted after any lines of therapy if patient ECOG PS was 0 or 1 .

\section{Comprehensive Genomic Profiling}

CGP was performed by Foundation Medicine CDx technology. The complete panel of genes analyzed in this study is shown in -Supplement Table S1 (online only). The turnaround time was 3 weeks. The analysis also included PDL1 expression by immunohistochemistry (Dako 22C3 platform). Microsatellite instability (MSI) was evaluated by genome-wide analysis of 95 microsatellite loci. This assay detected alterations in a total of 324 genes, using the Illumina HiSeq 4000 platform. Hybrid-capture-selected libraries were sequenced to high uniform depth (targeting $>500 \mathrm{X}$ median coverage with $>99 \%$ of exons at coverage $>\times 100$ ). Sequence data were processed using a customized analysis pipeline designed to detect all classes of genomic alterations, including base substitutions, indels, copy number alterations (amplifications and homozygous deletions), and selected genomic rearrangements (e.g., gene fusions) (-Supplement Table S1, online only).

\section{Treatment}

Patients were treated with the first-line chemotherapy: gemcitabine $1,000 \mathrm{mg} / \mathrm{m}^{2}$ and cisplatin $25 \mathrm{mg} / \mathrm{m}^{2}$ on day 1 and 8 every 3 weeks for three cycles, and response assessment was performed. For patients presenting with obstructive jaundice, obstruction was relieved by endoscopic retrograde cholangiopancreatography and stenting or percutaneous transhepatic biliary drainage wherever feasible. For responding patients (partial response or stable disease), three more cycles of the same regimen were given. In the second-line and subsequent-line settings, wherever target was detected, the patient received targeted therapy. Molecular targets were ERBB2, EBBB3, amplification and/or mutations, MET amplification, and PIK3 mutations. Toxicity data were collected, and response to therapy was assessed by clinical benefit and/or imaging and serological markers (CA19-9 and CEA). As per protocol, targeted therapy was allowed only in the second and subsequent line of therapy. Patients who had ERBB2 amplification received trastuzumab plus chemotherapy (nab-paclitaxel or FOLFOX). On trastuzumab progression, eligible patients received lapatinib and capecitabine. Patients with ERBB3 amplification received trastuzumab plus lapatinib, and ERBB3-mutated patients received lapatinib. MET amplification was treated with crizotinib $250 \mathrm{mg}$ BD. Everolimus was used in PIK3-mutated patients. Immunotherapy was not administered.

\section{Statistics}

Descriptive statistics was used to analyze the variables. To assess the association between variables, chi-square test and Fisher's exact test were used wherever needed. Survival analysis was performed using the Kaplan-Meier method. Log-rank test was used to evaluate the outcome differences between groups of patients. Cox regression analysis was used 
for univariate analysis. The significant univariate variables of value up to $p<0.10$ were considered for multivariate analysis using Cox regression proportional hazard analysis.

\section{Results}

A total of 50 patients were studied, and CGP information was available for all patients. Median age was 56.5 years (range: 26-83 years), with a male-to-female ratio of $1: 1.6$. Three patients underwent laparoscopic biopsies. Biopsy for liver lesion was performed in rest of the patients, and no major complication was observed. Two patients required observation for persistent pain for 24 hours. No fistula formation was observed. The targeted genomic landscape with percentages is shown in - Fig. 1. The targeted findings (ERBB2, ERBB3, MET, and PIK3 aberrations) were not frequent in patients aged $<60$ years as compared with those aged $>60$ years $(p=0.78)$. Details of patients, disease status, and response to treatment are given in - Table 1.

ERBB2 amplification was observed in nine (18\%) of the cases. ERBB3 aberrations were seen in five (10\%) cases, including two amplifications and three point mutations. MET amplification was seen in three patients. It was co-amplified with ERBB2 in one patient, and another had ERBB3 mutation along with MET amplification. Four patients received trastuzumab and chemotherapy in various lines of therapy. One patient showed partial response, two patients had progressive disease, and one patient had mixed response. One patient died before the next-generation sequencing (NGS) report was available, and one patient had sudden death not related to malignancy before the start of therapy.

Concurrent mutation and amplifications were seen at high rate. Of seven patients, two patients had concurrent ERBB2 mutation and amplification. These mutations were
S310F and V777L. One patient with S310F mutation did respond to lapatinib and continued with the same regimen. The point mutations in ERBB3 domain were V104L, G284R, and R426W. One patient of ERBB3 mutation maintained response to lapatinib beyond 12 weeks.

PIK3 mutations were seen in seven (14\%) cases. No concurrent PIK3 mutations were seen with ERBB2 and ERBB3 alterations. Three patients had PIK3CA H1047R mutation and two patients had PIK3CA-E545K mutation, highlighting recurrent genomic alterations in these domains.

TP53 gene abnormalities were present in $85 \%$ of cases. However, there were no recurrent genomic signatures in TP53. NF mutations were seen in five (10\%) patients, NF1 mutations in three patients, and NF2 mutations in two patients. Only one patient had fibroblast growth factor receptor (FGFR) 2 mutation, and one had FGFR3 amplification. Two patients were screened for germline mutation for PTEN loss and NF1 mutations by NGS. Both patients did not have germline abnormality.

PDL1 expression data were available for 35 patients, and it was $>=1 \%$ in $31 \%$ of cases. PDL1 expression ranged from 1 to $100 \%$ and did not show prognostic significance at a cutoff of $1 \%$. Tumor mutational burden (TMB) data were available for 43 patients. Median TMB was 5 mut/Mb, with a range of 1 to 14 mut/Mb. MSI $(n=43)$ was stable in all cases.

\section{Discussion}

A prospective study of NGS has not been conducted in GBC previously. Various retrospective series have been published in the GBC evaluating the targets by NGS. Biliary tract cancers comprise intrahepatic cholangiocarcinoma, extrahepatic cholangiocarcinoma, and GBCs. In this study, only GBC subset was studied, strengthening the literature for this subsite. Across all age ranges, actionability was found.

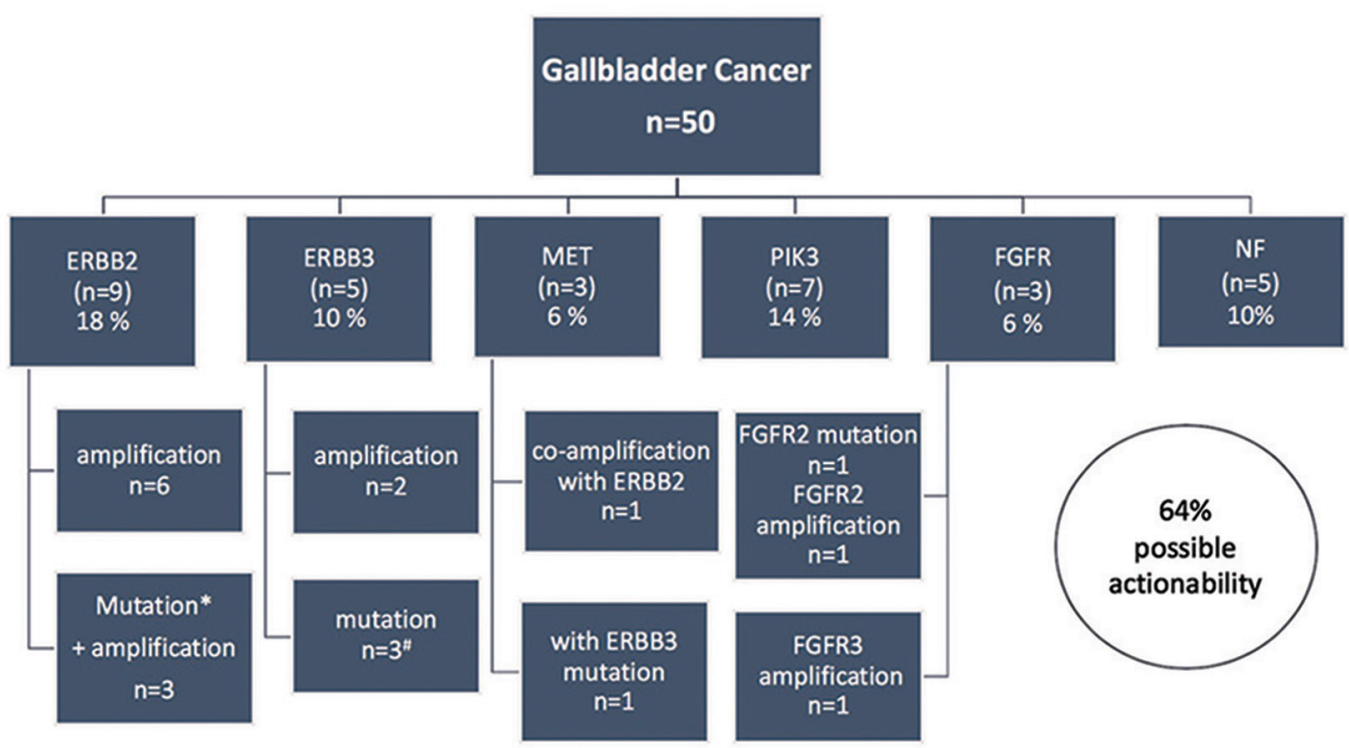

Fig. 1 Targeted genomic landscape in 50 patients of gallbladder cancer. * ERBB2 S310F, L869R, and V777L mutations were concurrently present with amplification. *ERBB3 mutations were V104L, G284R, and R426W. 
Genomic Landscape and Targeted Treatment of Gallbladder Cancer Patel et al. 77

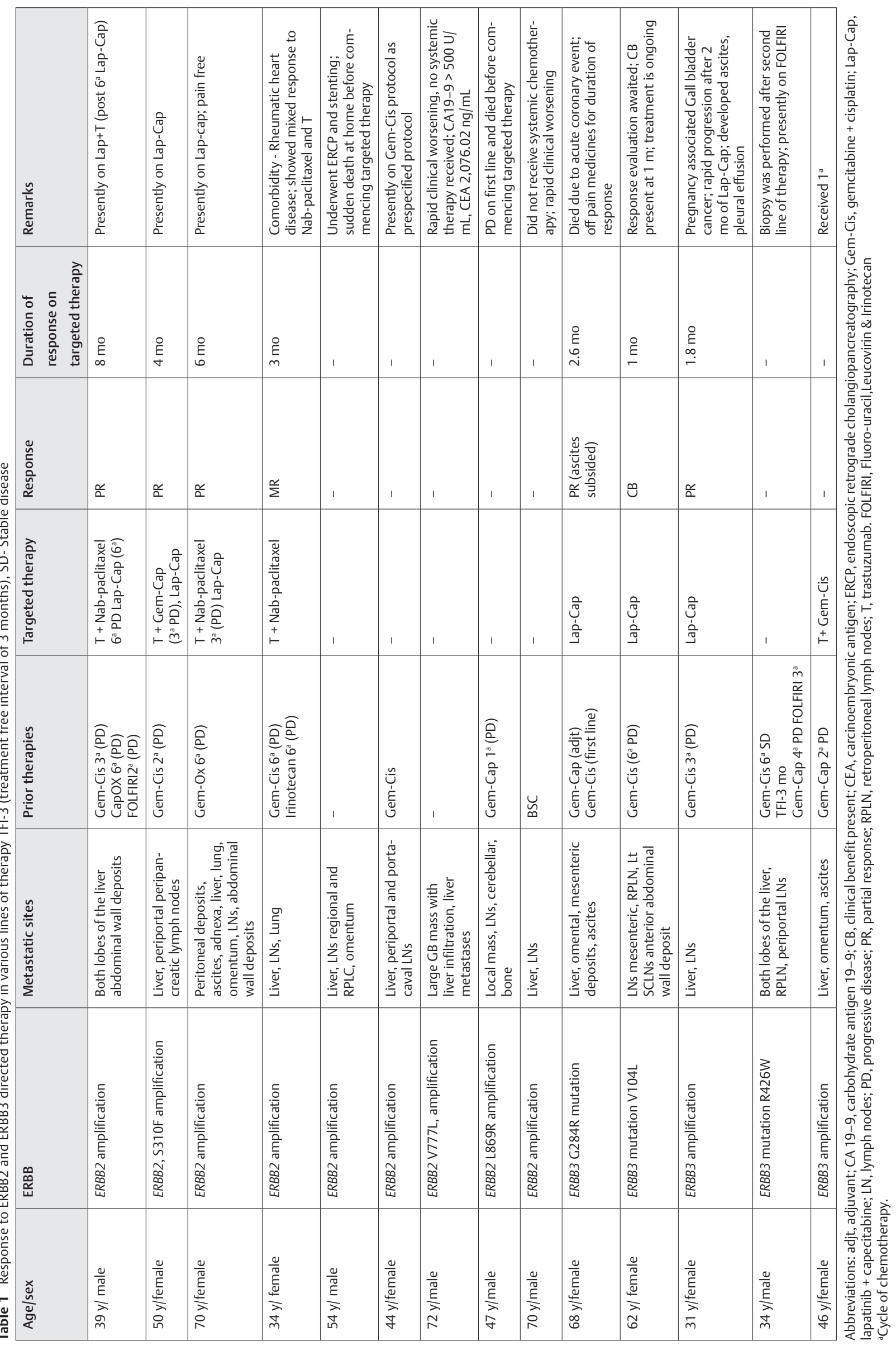


In a study by $\mathrm{Li}$ et al of whole-exome and targeted-gene sequencing in GBC, ERBB pathway was extensively mutated in $36.8 \%$ tissue samples. ${ }^{7}$ In this study, $16 \%$ had ERBB2 amplification. This study was limited by the absence of clinical data.

ERBB2, S310F, L869R, and V777L mutations were seen in three patients. These mutations are reported in other solid malignancies. They may pose resistance to trastuzumab.8-10 The patients with S310F mutation responded to lapatinib. In this ongoing study, we found promising responses to trastuzumab in the second-line therapy. The work on ERBB2-targeted therapy in biliary tract cancer was published by Javle et al. ${ }^{11}$ In this retrospective series, six cases of GBC were treated with trastuzumab, and responses were seen for short duration.

ERBB3 mutations were seen in three (7\%) patients, in whom encouraging responses to lapatinib (a pan-ERBB inhibitor) were seen. Li et al studied ERBB2/ERBB3 mutation and PDL1 expression in cell lines. ERBB2 and ERBB3 mutations were seen in 7 to $8 \%$ of GBC samples. ${ }^{12}$ We also found similar rates of mutations.

Two patients of ERBB2 amplification had coamplification with MET, which is not reported before in GBC. One patient with MET amplification received crizotinib; however, response lasted for 4 weeks. This highlights the importance of deeper understanding of the role of molecular pathogenesis of GBC and mechanisms of resistance for these pathways. Ratio of MET/CEP7 > 2.2 was suggested for the effectiveness of crizotinib in lung cancer trials. ${ }^{13}$ Future studies are warranted to explore the correlation between NGS and MET/CEP7 ratio on FISH (fluorescence in situ hybridization).

FGFR2 mutations, amplifications, and fusions have been reported in intrahepatic cholangiocarcinoma in $15 \%$ of patients with characteristics of indolent clinical course. ${ }^{14-16}$ Our study highlights the importance of being site-specific for biliary tract cancers. In this study, FGFR2 mutation-amplification was seen in one patient each. One patient had FGFR3 amplification. Recently, FGFR inhibitor was approved in urinary bladder carcinoma, and it is being studied in intrahepatic cholangiocarcinoma with early promising results. ${ }^{17,18}$

PIK3 mutations H1047R (three cases) and E545K (two cases) have not been reported before in GBC in prospective setting. These are possible targets for alpelisib, which received approval for metastatic breast cancer. The genomic signatures were similar to breast cancer. ${ }^{19,20}$

Previously, the percentage of actionability has been reported $^{21}$; however, these are retrospective in nature and carried bias. PDL1 expression > 1\% was seen in $31 \%$ of cases in our study. PDL1 expression by microarray technique is studied by Neyaz et al. ${ }^{22}$

TMB as a biomarker for immunotherapy has been studied in various malignancies, such as lung cancer, renal cell carcinoma, and head and neck cancer. TMB was analyzed by Yang et al in tissue samples of GBC, and reported mutational burden in $17 \%,{ }^{23}$ with a median TMB of $5 \mathrm{mu} / \mathrm{Mb}$, which is very much similar to our data. Their study was limited by nonavailability of clinical data, retrospective design, and two different cohorts of Chinese and American patients. We used the Foundation Medicine CDx platform, which is a validated tool for the use of immunotherapy.

MSI $(n=43)$ was stable in all cases. As the prevalence is $<5 \%$ for MSI, ${ }^{24}$ the use of it as an immunotherapy biomarker is of limited utility and requires future larger data.

\section{Strength of the Study}

The main strengths of this study are that GBC as a subset of biliary tract malignancy was studied and the role of precision therapy was explored in the second-line settings.

\section{Limitation of the Study}

A limitation of this study was that as a pilot work, the numbers of patients were less. The survival data and correlation with baseline variables will be published once data mature.

\section{Conclusion}

Role of personalized and precision medicine by CGP has expanded in GBC. Percentage of driver mutations differs by site in biliary tract malignancies. GBC is characterized preponderance of ERBB alterations (26\%), including ERBB2 and ERBB3 amplification and mutations. ERBB3 domain mutation can be targeted with lapatinib. Coamplifications and comutations are possible mechanisms of resistance in GBC for trastuzumab. Future studies on combination therapies with lapatinib or pertuzumab are needed. FGFR2 mutation and amplification are rare as compared with intrahepatic cholangiocarcinoma. PIK3 mutations were present in 14\% of cases with recurrent genomic signatures. Outcomes with targeted therapies in the second-line setting will be published once data mature.

\section{Funding}

FMI (Foundation Medicine, Inc) supported evaluation samples for this study.

\section{Conflicts of Interest}

There are no conflicts of interest.

\section{Acknowledgments}

We sincerely thank our patients for participating in this study. We extend our thanks to Prof Atul Sharma for source of inspiration on Gallbladder cancer research and also thank Dr Prashant Mehta, Dr C K Das \& Dr Vineet Govinda Gupta for their valuable inputs.

\section{References}

1 Randi G, Franceschi S, La Vecchia C. Gallbladder cancer worldwide: geographical distribution and risk factors. Int J Cancer 2006;118(7):1591-1602

2 Malhotra RK, Manoharan N, Shukla NK, Rath GK. Gallbladder cancer incidence in Delhi urban: A 25-year trend analysis. Indian J Cancer 2017;54(4):673-677 
3 Shukla HS, Sirohi B, Behari A, et al. Indian Council of Medical Research consensus document for the management of gall bladder cancer. Indian J Med Paediatr Oncol 2015;36(2):79-84

4 Valle JW, Furuse J, Jitlal M, et al. Cisplatin and gemcitabine for advanced biliary tract cancer: a meta-analysis of two randomised trials. Ann Oncol 2014;25(2):391-398

5 Lindnér P, Holmberg E, Hafström L. Gallbladder cancer - no improvement in survival over time in a Swedish population. Acta Oncol 2018;57(11):1482-1489

6 ECOG-ACRIN. ECOG Performance Status. Available at: http:// ecog-acrin.org/resources/ecog-performance-status. Accessed December 28, 2016

7 Li M, Zhang Z, Li X, et al. Whole-exome and targeted gene sequencing of gallbladder carcinoma identifies recurrent mutations in the ErbB pathway. Nat Genet 2014;46(8):872-876

8 Mishra R, Hanker AB, Garrett JT. Genomic alterations of ERBB receptors in cancer: clinical implications. Oncotarget 2017; 8(69):114371-114392

9 Subramanian J, Katta A, Masood A, Vudem DR, Kancha RK. Emergence of $E R B B 2$ mutation as a biomarker and an actionable target in solid cancers. Oncologist 2019;24(12):e1303-e1314

10 Yi Z, Ma F, Guan Y, et al. Impact of HER2 mutation status on personalized molecular targeted therapy in advanced breast cancers. J Clin Oncol 2018;36(Suppl 15):1039

11 Javle M, Churi C, Kang HC, et al. HER2/neu-directed therapy for biliary tract cancer. J Hematol Oncol 2015;8:58

12 Li M, Liu F, Zhang F, et al. Genomic ERBB2/ERBB3 mutations promote PD-L1-mediated immune escape in gallbladder cancer: a whole-exome sequencing analysis. Gut 2019;68(6):1024-1033

13 Kawakami H, Okamoto I, Okamoto W, Tanizaki J, Nakagawa K, Nishio K. Targeting MET amplification as a new oncogenic driver. Cancers (Basel) 2014;6(3):1540-1552

14 Jain A, Borad MJ, Kelley RK, et al. Cholangiocarcinoma with FGFR genetic aberrations: a unique clinical phenotype. JCO Precision Oncol 2018;2:1-12
15 Ross JS, Wang K, Gay L, et al. New routes to targeted therapy of intrahepatic cholangiocarcinomas revealed by next-generation sequencing. Oncologist 2014;19(3):235-242

16 Graham RP, Barr Fritcher EG, Pestova E, et al. Fibroblast growth factor receptor 2 translocations in intrahepatic cholangiocarcinoma. Hum Pathol 2014;45(8):1630-1638

17 Javle M, Lowery M, Shroff RT, et al. Phase II study of BGJ398 in patients with FGFR-altered advanced cholangiocarcinoma. J Clin Oncol 2018;36(3):276-282

18 Food and Drug Administration. FDA approves first targeted therapy for metastatic bladder cancer. Available at: https:// www.fda.gov/news-events/press-announcements/fda-approves-first-targeted-therapy-metastatic-bladder-cancer. Accessed August 21, 2019

19 André F, Ciruelos E, Rubovszky G, et al; SOLAR-1 Study Group. Alpelisib for PIK3CA-Mutated, Hormone Receptor-Positive Advanced Breast Cancer. NEngl J Med 2019;380(20):1929-1940

20 Commissioner of the FDA approves first PI3K Inhibitor for Breast Cancer. Available at: https://www.fda.gov/newsevents/press-announcements/fda-approves-first-pi3k-inhibitor-breast-cancer. Accessed February 5, 2020

21 Lee H, Ross JS. The potential role of comprehensive genomic profiling to guide targeted therapy for patients with biliary cancer. Therap Adv Gastroenterol 2017;10(6):507-520

22 Neyaz A, Husain N, Kumari S, et al. Clinical relevance of PD-L1 expression in gallbladder cancer: a potential target for therapy. Histopathology 2018;73(4):622-633

23 Yang P, Javle M, Pang F, et al. Somatic genetic aberrations in gallbladder cancer: comparison between Chinese and US patients. Hepatobiliary Surg Nutr 2019;8(6):604-614

24 Goeppert B, Roessler S, Renner M, et al. Low frequency of mismatch repair deficiency in gallbladder cancer. Diagn Pathol 2019;14(1):36 